been long affected, and the other only showed signs of commencing dísease. It was not an objection to the operation to leave some of the diseased structures behind, as Mr. Lucas's case showed.-Mr. GoLdING BIRD was glad Mr. Lucas had reminded him of the morphia. When the man was returned to bed he became very restless, and then he gave him a quarter of a grain of morphia subcutaneously. Although the man died with small pupils, he felt sure the morphia was not the cause of death. He did not remove the whole rib, but only the middle of it, and that subperiosteally. The focus of disease in the prostate was not larger than a pea, and the other kidney was found quite healthy.

The Society then adjourned.

\section{MEDICAL SOCIETY OF LONDON.}

Extirpation of Myeloid Sarcoma and Melanotic Sarcoma without Recurrence. - Treatment of Chronic Dysentery by Enemata of Nitrate of Silver.-Atrophy of the Brain in Imbeciles.

A MeETing of this Society was held on March 20th, the chair zbeing taken by the President, Mr. Francrs Masor, who exhibited a man aged sixty-eight, from whom $\mathrm{Mr}$. Mason, in 1874, had removed a myeloid sarcoma of the septum nasi in three operations, the first by the polypus forceps; the second, three weeks later, by excision through the side of the nostril; and the third, three months afterwards, by reopening the former cicatrix and dividing the upper lip in the median line, and a portion of the inner surface of the lip inwardly; the growth was also excised. There had been no recurrence. Mr. Mason also mentioned a case of melanotic sarcoma of the right cheek, operated on in 1872. The submaxillary glands were so extensively involved that it was found impossible to remove all the disease. There was no recurrence of the growth, death taking place in 1880 from some other cause. - Mr. BUTLIN would not draw too sweeping conclusions from such cases, many of which gave disappointing results, varying with the site and the tissue in which the tumour occurred. Central sarcoma of the bone, like the first related, was much less malignant than periostea sarcoma. The second case was not parallel to the first; the glandular implication showed its carcinomatous nature Instances have been known of enlarged glands subsiding after removal of malignant disease of the tongue; in some cases the injury done by the operation may set up inflammatory processes in the glands of salutary tendency.

Dr. S. MACKENzIE read a paper on the Treatment of Chronic Dysentery by Voluminous Enemata containing nitrate of silver (the paper will be publishea in THe LANCET). - The PRESIDENT asked whether the author recommended washing out of the bowel before giving the enema.-Dr. Cullimore approved the method; he had used injections containing nitric acid. In a case treated in Burmah, of two years' standing, large injections of warm water were successful after the failure of other measures. Fried fish was of value as diet, and he had found good results from benzoin in acute dysentery.-Dr. RoGERs had found injections of charcoal and opium, with red gum given by the mouth, of great service. - Dr. DE HAVILLAND HALI asked whether the patients were urged to retain the enema He had tried this treatment with children, but with only moderate success, and had found extract of bael and con fection of bael of service.-Dr. GILBART SMITH asked how long should the enema be retained, and whether the injection of perchloride of iron, retained by means of a sponge would not be safer? It was a plan he had found to succeed in the diarrhoea of phthisis.-Dr. MACKENzIE, in reply, said he washed out the bowel only when scybala were present; that he spoke only of chrouic dysentery; that in most cases the injection is returned at once; and the afterinjection of salt, as practised in two of his cases, was unnecessary. The use of a sponge might incite harmful peristalsis.

Dr. FLETCHEI BEACH read a paper on Atrophy of the Brain in Imbeciles. Two forms are spoken of by writers. In the one there is incomplete development; in the other loss of nerve tissue previously present. Microcephalic imbeciles are examples of the first class. Microcephaly may be general or partial; some portions of brain may be too small or altogether absent; he had seen convolutions of certain parts much reduced in size from non-development, the remaining portion being too large or normal. The occipital lobes are often arrested in growth in microcephalic cases, and the corpus callosum may be shortened posteriorily or be absent. Usually, however, microcephaly is general, and the defect consists in the smallness of the hemispheres. In two cases related by him at the Congress last year the brains weighed $7 \mathrm{oz}$. and $20 \frac{1}{2} \mathrm{oz}$. respectively, and he had seen others since weighing $20 \frac{1}{4} \mathrm{oz}$. and $24 \frac{1}{2} \mathrm{oz}$. The cerebellum in microcephaly is relatively larger than normal, being often in the proportion of 1 to 3 or 1 to 4 , instead of 1 to 8 . Illustrative cases were given, and the fact that the brain tissue suffered in quality as well as quantity was pointed out, microscopical specimens showing deficiency in cell process and protoplasm. The second form of atrophy appeared in various forms ; the most interesting being atrophy of one side of the brain with coexistent atrophy of the limbs on the opposite side of the body. This kind of disease seemed to be caused by chronic meningitis or inflanmatory processes in the cortex before or after birth. Cases were related, the usual characteristics being thickness of the cranium, opacity and thickness of the membranes, effusion of serum into the snbarachnoid sface and sometimes into the ventricles, with atrophy of one hemisphere, including the corpus striatum and thalamus, and atrophy of the cerebellum and cord of the opposite side.The PRESIDENT asked as to the influence of consanguinity, and whether in these cases the palate was arched, or the thy roid enlarged. Had the use of forceps during delivery any share in their causation?-Dr. GILBART SMITH had seen two cases of deficiency of one side of the brain, with thinning of the skull. Was pressure of the skull tried as a means of diagnosis?-Dr. S. MACKENZIE said that the practical point was the mode of causation shown by Dr. Beach to consist in inflammation of the vascular parts of the encephalon, causing atrophy of the deeper structures. The stress of the lesion falling on the hinder parts of the brain, the seats of higher faculties, which are late in attaining their full developinent, therefore suffer more from causes originating after birth than the earlier developed parts. - Dr. BEACH, in reply, said that only two per cent. of cases of imbecility are accounted for by cousin-marriages. Though arching of palate is found in these cases as well as in sane people, its presence may be taken to prove the congenital nature of the state. Goitre is not always an accompaniment; in some cases fatty tumours occurred in the neck with or without goitre. Open sutures are found in some cases; therefore synostosis is not the cause of the lesion. The use of the forceps was not shown by recent observation to be a cause of imbegility a prolonged labour was more 'dangerous. He had not seen any cases with thinning of the skull.

\section{THE ST. GOTHARD PARASITE IN INDIA.} To the Editor of THE LANCET.

SIR,-By this mail Professor J. F. P. McConnell informs me that he has discovered Dochmius cluodenalis in Calcutta, and it seems that the parasite is by no means uncommon in India. Although there will be no astonishment at this announcement, yet the find adds largely to our knowledge of the geographical distribution of this injurious parasite. Professor McConnell states that the worm is by no means confined to the upper division of the small intestines; on the contrary, the majority of the specimens were removed from the mucous membrane of the jejunum. They were firmly fixed to the gut. As Professor MicConnell found the parasites in a large number of post-mortems, in cases where the victims, who are natives, died of many different diseases, he does not feel justified in stating that they were the sole cause of death in any case. In many patients he admits that anæmia was the prevailing feature, but attributes this rather to dysenteric and malarial complications. His inves. tigations supply many other interesting details, which will doultless be published in due course.

March, 18s: I am, Sir, your obedient servant,

T. SPHNCER COBEOLD.

Charing-cross Hospital. - The Duke of Edinburgh, President of the Charingacross Hospital, will preside at the festival dinner in aid of the funds of the institution, to be beld on April 19th at Willis's Rooms. 


\section{THE LANCET.}

LONDON: SATURDAY, APRIL 1, 1882.

THe important meeting held at the College of Physicians on Tuesday last, to found an Association for the Advancement of Medicine by Research, is the outcome of two recent events-the meeting of the International Medical Congress, at which the absolute necessity of the experimental method for the advancement of medical knowledge was made so abundantly apparent; and the prosecution of Dr. FERRIER, which demonstrated the unreasoning persecution to which British experimenters are subjected. Months have elapsed since the movement then started took shape, but the time has been busily and well spent by a few earnest men, and the result is the formation of what ought to be the most important Association for the Advancement of Medicine ever founded. It is impossible to forecast the work that will be done by it, although we may form some estimate of what it may endeavour to accomplish. And when we attempt this estimate, and try to ascertain what the formation of such an Association really means, we are at once struck with the difference between it and any of the existing medical societies and associations. It does not exist for any political, social, or purely educational purposes, nor for any direct advantage of medical men themselves, but purely and simply for the advance of medicine by research. It may be asked, What can an association do to promote scientific research? The actual work of research must be done by individual workers. True, but their efforts may be stimulated, guided, or restrained by the co-operation of a body of men specially fitted to appreciate their aims and advise them in their procedure. And while the Association may be able to do much by its direct influence upon the profession, it may do more by its influence upon the public. By exaggerated statements, and fictitious appeals to an excellent sentiment, a false feeling has been excited in some quarters which renders medical research in many cases diffcult, in some even impossible. A thoroughly representative association, such as this is, cannot but have great weight with the more intelligent part of the general public.

There are some features in the Association which are of specially hopeful omen. The first of these is the co-operation of men distinguished in all branches of science with workers in medicine. We are all willing enough to admit the close dependence of medical science upon other sciences, but never before has there been such a practical acknowledgment of the fact. This must tend to widen the scope of researches undertaken by, or carried out under the auspices of, the Association; and it will be a means of communication between physicians and other men of science that cannot fail to widen the interest each feels in the work of the other, and to bring to bear upon medical problems the rich stores of information gathered in other fields. It is also noteworthy that this Association is formed for the alvancement of medicine by researich, -not by research and observation, still less by observation alone. This is a distinctly new departure. It is a formal declaration that it is by research that we may now hope to advance our science. While not ignoring the fruits of observation, the formation of this Association is a practical assertion that there is a better method from which greater results are to be expected, and which must be deliberately and persistently pursued. Not less encouraging is the broad basis on which the Association is to work. It is not to be interested in one form of research only-for instance, it is not to protect vivisectors alone; but its aim is to assist and stimulate every kind of research, physical, chemical, or vital, which has any bearing upon medicine. The best evidence that much is to be hoped for from this Association is the spirit that evidently animates the leaders of the movement. There is no sign of vindictiveness, of opposition to existing laws; it is emphatically not an anti-society, and not marked by any narrow party spirit. The profession is not asked to agitate for the repeal or amendment of any law. Existing laws are to be com. plied with and used, and their effect upon scientific research carefully watched and exactly defined.

The work undertaken by this Association is as difficult and delicate as it is important, and we would echo every word uttered by Sir W. JENNER and Sir RISDON BENNETT on this point. The Council of the Association, upon whom all this labour will devolve, will need all the discretion which even age can bring to enable it to steer clear of the many dangers in its path. We may expect to see its first efforts devoted to the publication of carefully prepared information on the need of the experimental method, its advantages, the benefits that have already resulted from it, and the work yet awaiting it. It is no easy matter to accomplish this task in a worthy manner. But from the tone of the meeting of Tuesday last it is evident that the Council may rely upon the aid of the very best men, not only among ourselves, but in all branches of science. It is hoped that Sir JAMEs PAGET will be appointed by the Council its vice-chairman, and, as this officer is by the rules of the Association eligible for re-election, it is desired to secure his services in a somewhat permanent way. If this arrangement can be carried through it will be an important step towards securing the success of the Association.

We must refer to the account contained in another column of the meeting held at the College of Physicians for the full particulars of the Association, and will now only claim for it the sympathy and co-operation of the whole body of the profession.

ThE residence of H.R.H. the Duke of ConNAUGHT at Bagshot Park furnishes an additional illustration of the peculiarities which attach to British mansions as a rule, whether big or little, royal or plebeian, and the building thereof. Happily this illustration is shorn of the disastrous consequences which have too frequently accompanied previous illustrations, and of which the most melancholy example rests with the Royal Family. Designers, constructors, and pro. prietors, with few exceptions, hold to the traditional belief that a house is built to live in, and so long as it pleases the eye, shuts out wind and weather, ard meets the convenience and comfort of the occupier, there is nothing more to be said. The conception that the house may be so constructed as to 